\title{
Evaluation of Pattern Recognition Techniques in Response to Cardiac Resynchronization Therapy (CRT)
}

\author{
Mohammad Nejadeh \\ Department of Computer Engineering, Islamic Azad University, Rasht Branch, Rasht, Iran \\ m.nejadeh@fshiau.ac.ir \\ Peyman Bayat* \\ Department of Computer Engineering, Islamic Azad University, Rasht Branch, Rasht, Iran \\ bayat@iaurasht.ac.ir \\ Jalal Kheirkhah \\ Department of Cardiology, Healthy Heart Research Center, School of Medicine, Guilan university of Medical Sciences, Rasht, Iran \\ kheirkhah@gums.ac.ir \\ Hassan Moladoust \\ Healthy Heart Research Center, School of Medicine, Guilan university of Medical Sciences, Rasht, Iran. \\ hmoladoust@gums.ac.ir
}

Received: 30/Aug/2020 Revised: 05/Oct/2020 Accepted: 08/Nov/2020

\begin{abstract}
Cardiac resynchronization therapy (CRT) improves cardiac function in patients with heart failure (HF), and the result of this treatment is decrease in death rate and improving quality of life for patients. This research is aimed at predicting CRT response for the prognosis of patients with heart failure under CRT. According to international instructions, in the case of approval of QRS prolongation and decrease in ejection fraction (EF), the patient is recognized as a candidate of implanting recognition device. However, regarding many intervening and effective factors, decision making can be done based on more variables. Computer-based decision-making systems especially machine learning (ML) are considered as a promising method regarding their significant background in medical prediction. Collective intelligence approaches such as particles swarm optimization (PSO) algorithm are used for determining the priorities of medical decision-making variables. This investigation was done on 209 patients and the data was collected over 12 months. In HESHMAT CRT center, 17.7\% of patients did not respond to treatment. Recognizing the dominant parameters through combining machine recognition and physician's viewpoint, and introducing back-propagation of error neural network algorithm in order to decrease classification error are the most important achievements of this research. In this research, an analytical set of individual, clinical, and laboratory variables, echocardiography, and electrocardiography (ECG) are proposed with patients' response to CRT. Prediction of the response after CRT becomes possible by the support of a set of tools, algorithms, and variables.
\end{abstract}

Keywords: Cardiac resynchronization therapy; Neural Networks; Particle swarm optimization; HESHMAT_CRT dataset; Machine Learning.

\section{1- Introduction}

The unsuccessful function of the heart can result from different causes such as vascular occlusion and high blood pressure, and this problem is referred to as heart failure (HF). In this condition, cardiac muscle is not able to pump enough blood in the body [1]. Arrhythmia is a disorder of heartbeat rhythm. This disorder makes the heart unable to effectively pump blood all over the body and patients with arrhythmia usually experience the symptoms of rapid and slow heartbeat [2]. Patients with HF and arrhythmia may be appropriate candidates for CRT [3]. In addition to improvement of cardiac output in a short time, it helps to increase patients' lifetime and decrease of $\mathrm{HF}$ hospitalization cases [4]. Doctors' suggestions may be different depending on their observation of the patient's condition, and the results of selecting candidate patients in the past do not seem to be good.

Many risks can occur during the CRT process and there might be various side effects [5]. The majority of patients (35-40\%) do not respond to CRT due to unknown causes [6], and it has a negative effect on function and efficiency of this treatment for HF patients [7]. The research idea is about proposing a new feature vector and creating a pattern detection solution for identifying appropriate candidates for CRT with high accuracy. It can increase the rate of response to CRT and practically decrease the rate

* Corresponding Author 
of no response. Machine learning is a powerful computation method that can provide the possibility of a better description of effective parameters. Decision support tools are developed for the prediction of clinical results and create better decisions for similar cases of patients [8]. The possibility of prediction based on intelligent algorithms is a step forward to identifying candidates for CRT. These operations are non-invasive and used for optimizing the treatment process, and they can significantly help doctors provided that they are properly operated [9]. After the implant, there are very few and sometimes impossible ways to overcome the cases of no response [10]. The process of machine learning-based prediction requires using the collected medical data [11]. In order to create analysis features in most of the datasets relevant to the research topic, three methods are used including (1) extracting feature from cardiac imaging [12], (2) a combination of the features extracted from ECG and the patient's laboratory data [13,8], and (3) combining an important parameter such as (NYHA) classification function and analysis of medical examinations with other techniques such as natural language processing (NLP) [14], or using QRS variable international suggestions [15]. The research data set consists of 67 independent attributes and 1 dependent attribute. Independent characteristics were obtained according to the patient's medical record, laboratory results, imaging, and independent characteristics were obtained according to postoperative results.

The main purpose of this study is to introduce a set of cardiac synchronization therapy data, identify patients suitable for treatment with machine learning algorithms, determine the role of important features and their priorities. The determination of effective features by the medical doctor is collected from the feature vector, the prioritization of features by intelligent algorithms, and their effective role in obtaining a better classification answer are discussed.

In the following, related works on the analysis of CRT patients are reviewed. All the researches have been performed aimed at proposing a method for making HF treatment efficient. Various researches have addressed the results by predictors $[16,8,10,17]$. The research [18] investigated the relationship between the variables aimed at improving prediction of CRT response, and the research [19] investigated the analysis of data after optimizing the device and setting the device in different locations. In the researches $[20,9,21,22]$, the effect of other cardiac factors in patients under CRT was investigated. Some studies such as [23] provided statistical reports of the related works and so, helped to prepare a new dataset. In spite of the same instructions, [24] indicates that CRT is not still fully used everywhere. Regarding the datasets of previous works, we can mention [21] with 25 features and 54 samples, [25] with 18 features and 428 samples, [26] with 25 features and 679 samples, [27] with 32 features and 23 samples, and [8] with 45 features and 595 samples for predicting CRT condition. The three studies of $[8,10$, 27] used machine learning for selecting CRT candidate patients. In the research [8], the effective variables were analyzed based on four quartiles. These charts were composed of two variables of LBBB and QRS. The variable QRS was defined at two states of QRS $\geq 150 \mathrm{~ms}$ and QRS $<150 \mathrm{~ms}$, and LBBB was defined in a binary manner. Efficiency of the random forest (RF) algorithm was evaluated by reviewing the classifiers. Since the collected data including class variables have different levels, and determination of importance and priority of features by RF algorithm is not reliable, increasing and decreasing the samples in [8] resulted in many changes in classification error. On the other hand, with the existence of unknown parameters in the provided dataset that can play the role of noise, using the RF algorithm will cause over-fitting (OF). However, the research method classifies QRS variable in three levels of short, medium, and long. Back-propagation of error neural network model is prioritized is used for decreasing implementation classification error and the variables selected by the doctor are prioritized by PSO algorithm. The results will show that neural models such as backpropagation of error neural network and Learning Vector Quantization compared to other machine learning algorithms such as Naive Bayes (NB), support vector machine (SVM), random forest (RF), k-Nearest Neighbors has higher efficiency on research data sets. Also, 7 out of 9 features by the medical doctor were prioritized.

The following sections of the paper are organized as the following. First, the general properties of the research dataset, features, and primary pre-processing are described by transformation and discretization, and the research method is explained for the implementation of the dataset. The results are discussed by evaluating the algorithms and conclusion of the research is presented. Also, some suggestions are proposed for developing decision making systems for working on the present dataset. 


\section{2- Medical dataset}

The data was collected by individual, laboratory, clinical, electrocardiogram, and echocardiography. The limited number of surgeries in treatment centers (Heshmat Hospital of Rasht), no access to the patients after the surgery, the need to a specialist for reviewing the medical files, and incompleteness of the files are some of the data collection constraints. Feature extraction was done by using a questionnaire before and after implanting CRT device, medical examinations, blood sampling, ECG observations, and echocardiography. Table (1) presents a full description of the extracted features. The research dataset includes 209 cardiac patients gotten CRT-D implant. 38 people did not respond to the treatment and 171 people indicated a positive response. Some of the personal information of patients was excluded for security considerations and also, some of the deficient and calculable were removed. The main features and their range are specified in Table (1).

Table 1: The features extracted for prediction of CRT response

\begin{tabular}{|c|c|c|}
\hline Type & Feature Name & Range \\
\hline \multirow{6}{*}{ 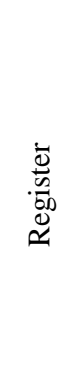 } & File Number & - \\
\hline & Patient Name & - \\
\hline & Patient contact number & - \\
\hline & $\begin{array}{c}\text { Companion patient 's contact } \\
\text { number }\end{array}$ & - \\
\hline & Surgical Date & - \\
\hline & Doctor's name & - \\
\hline \multirow{11}{*}{ 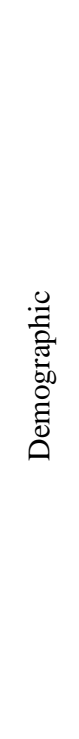 } & Age & $25-86$ \\
\hline & Sex & $\begin{array}{l}1=\text { Male, } \\
2=\text { Female }\end{array}$ \\
\hline & DM (Diabetes Mellitus) & Yes, No \\
\hline & HTN (Hyper Tension) & Yes, No \\
\hline & Current Smoker & Yes, No \\
\hline & Ex-Smoker & Yes, No \\
\hline & FH (Family History) & Yes, No \\
\hline & CRF (Chronic Renal Failure) & Yes, No \\
\hline & CVA (Cerebrovascular Accident) & Yes, No \\
\hline & $\begin{array}{c}\text { CABG(Coronary artery bypass } \\
\text { surgery) }\end{array}$ & Yes, No \\
\hline & Airway Disease & Yes, No \\
\hline
\end{tabular}

\begin{tabular}{|c|c|c|}
\hline Type & Feature Name & Range \\
\hline & Thyroid Disease & Yes, No \\
\hline & CHF (Congestive Heart Failure) & Yes, No \\
\hline & DLP (Dyslipidemia) & Yes, No \\
\hline \multirow{13}{*}{ 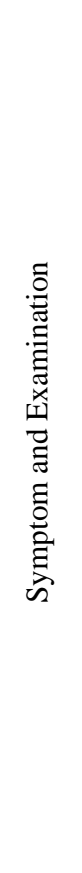 } & BP (Blood Pressure: mmHg) & $90-190$ \\
\hline & Edema & Yes, No \\
\hline & Weak peripheral pulse & Yes, No \\
\hline & Lung Rales & Yes, No \\
\hline & Systolic murmur & Yes, No \\
\hline & Diastolic murmur & Yes, No \\
\hline & Typical Chest Pain & Yes, No \\
\hline & Dyspnea & Yes, No \\
\hline & $\begin{array}{c}\text { NYHA (New York Heart } \\
\text { Association Function Class) }\end{array}$ & $1,2,3,4$ \\
\hline & Atypical & Yes, No \\
\hline & Nonanginal CP & Yes, No \\
\hline & $\begin{array}{c}\text { Exertional CP (Exertional Chest } \\
\text { Pain) }\end{array}$ & Yes, No \\
\hline & $\begin{array}{c}\text { Low Th Ang (low Threshold } \\
\text { angina) }\end{array}$ & Yes, No \\
\hline \multirow{15}{*}{ 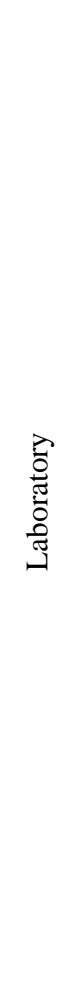 } & FBS (Fasting Blood Sugar) (mg/dl) & $62-400$ \\
\hline & $\mathrm{Cr}$ (creatine) $(\mathrm{mg} / \mathrm{dl})$ & $0.5-2.2$ \\
\hline & TG (Triglyceride) (mg/dl) & $37-1050$ \\
\hline & Total chol & - \\
\hline & $\begin{array}{l}\text { LDL (Low density lipoprotein) } \\
(\mathrm{mg} / \mathrm{dl})\end{array}$ & $18-232$ \\
\hline & $\begin{array}{l}\text { HDL (High density lipoprotein) } \\
(\mathrm{mg} / \mathrm{dl})\end{array}$ & $15-111$ \\
\hline & $\begin{array}{l}\text { BUN (Blood Urea Nitrogen) } \\
(\mathrm{mg} / \mathrm{dl})\end{array}$ & $6-52$ \\
\hline & $\begin{array}{l}\text { ESR (Erythrocyte Sedimentation } \\
\text { rate })(\mathrm{mm} / \mathrm{h})\end{array}$ & $1-90$ \\
\hline & HB (Hemoglobin) (g/dl) & $8.9-17.6$ \\
\hline & $\mathrm{K}$ (Potassium) (mEq/lit) & $3.0-6.6$ \\
\hline & $\mathrm{Na}$ (Sodium) (mEq/lit) & $128-156$ \\
\hline & $\begin{array}{l}\text { WBC (White Blood Cell) } \\
(\text { cells } / \mathrm{ml})\end{array}$ & $3700-18000$ \\
\hline & CKMB & - \\
\hline & Troponin & - \\
\hline & Lymph (Lymphocyte) (\%) & $7-60$ \\
\hline
\end{tabular}




\begin{tabular}{|c|c|c|}
\hline Type & Feature Name & Range \\
\hline & Neut (Neutrophil) (\%) & $32-89$ \\
\hline & PLT (Platelet) $(1000 / \mathrm{ml})$ & $25-742$ \\
\hline \multirow{16}{*}{$\begin{array}{l}0 \\
\frac{\pi}{4} \\
\infty \\
0 \\
0 \\
0\end{array}$} & Rhythm & Sin, $\mathrm{AF}$ \\
\hline & Q Wave & Yes, No \\
\hline & HR & $50-110$ \\
\hline & PR Interval & - \\
\hline & QT Interval & - \\
\hline & QRS Duration & - \\
\hline & Fragment & Yes, No \\
\hline & Number of fragment & $0-8$ \\
\hline & ST Elevation & Yes, No \\
\hline & ST Depression & Yes, No \\
\hline & $\mathrm{T}$ inversion & Yes, No \\
\hline & $\begin{array}{l}\text { LVH (Left Ventricular } \\
\text { Hypertrophy) }\end{array}$ & Yes, No \\
\hline & $\begin{array}{l}\text { Poor R Progression(Poor R Wave } \\
\text { Progression) }\end{array}$ & Yes, No \\
\hline & $\begin{array}{l}\mathrm{LV} \mathrm{EF} \text { (left ventricular ejection } \\
\text { fraction) }(\%)\end{array}$ & Number \\
\hline & AI (Normal,mild,moderate,severe) & $1-4$ \\
\hline & $\begin{array}{c}\text { MR } \\
\text { (Normal,mild,moderate,severe) }\end{array}$ & $1-4$ \\
\hline & Device Type & - \\
\hline Result & CRT Response & Yes, No \\
\hline
\end{tabular}

In the present dataset, there are many features that provide useful information by discretization. Discretization is done based on standard medical ranges and other methods such as class entropy, bucketing, range frequency-varying domain, numbers distance determination, and clustering. Table (2) presents the method of discretization of general and laboratory features. General features refer to the features related to symptoms, examination, and demography. The most important variables of the final feature vector for prediction of CRT response are resulted from ECG and the patient's echocardiography.
Table 2: Discretization of general and laboratory features

\begin{tabular}{|c|c|c|c|}
\hline Feature & Low & Normal & High \\
\hline $\mathrm{Cr} 2$ & $\mathrm{Cr}<0.7$ & $0.7 \leq \mathrm{Cr} \leq 1.5$ & $\mathrm{Cr}>1.5$ \\
\hline FBS2 & FBS $<70$ & $70 \leq \mathrm{FBS} \leq 105$ & FBS > 105 \\
\hline LDL2 & & $\mathrm{LDL} \leq 130$ & LDL > 130 \\
\hline HDL2 & $\mathrm{HDL}<35$ & $\mathrm{HDL} \geq 35$ & - \\
\hline BUN2 & BUN $<7$ & $7 \leq \mathrm{BUN} \leq 20$ & BUN > 20 \\
\hline ESR2 & - & $\begin{array}{c}\text { if male \& } \\
\mathrm{ESR} \leq \mathrm{Age} / 2 \\
\text { or } \\
\text { if female \& } \\
\mathrm{ESR} \leq \text { Age } / 2+5\end{array}$ & $\begin{array}{c}\text { if male \& } \\
\text { ESR }>\text { Age } / 2 \\
\text { or } \\
\text { if female \& } \\
\text { ESR > } \\
\text { Age } / 2+5\end{array}$ \\
\hline $\mathrm{Hb} 2$ & $\begin{array}{c}\text { if male \& } \\
\mathrm{Hb}<14 \\
\text { Or If female } \\
\& \\
\mathrm{Hb}<12.5\end{array}$ & $\begin{array}{c}\text { if male \& } \\
14 \leq \mathrm{Hb} \leq 17 \\
\text { Or if female \& } \\
12.5 \leq \mathrm{Hb} \leq 15\end{array}$ & $\begin{array}{c}\text { if male \& } \\
\mathrm{Hb}>17 \\
\text { Or if female } \\
\& \\
\mathrm{Hb}>15\end{array}$ \\
\hline K2 & $\mathrm{K}<3.8$ & $3.8 \leq \mathrm{K} \leq 5.6$ & $\mathrm{~K}>5.6$ \\
\hline $\mathrm{Na} 2$ & $\mathrm{Na}<136$ & $136 \leq \mathrm{Na} \leq 146$ & $\mathrm{Na}>146$ \\
\hline WBC2 & $\begin{array}{c}\text { WBC }< \\
4000\end{array}$ & $\begin{array}{c}4000 \leq \mathrm{WBC} \leq \\
11000\end{array}$ & $\begin{array}{l}\text { WBC > } \\
11000\end{array}$ \\
\hline PLT2 & PLT $<150$ & $150 \leq \mathrm{PLT} \leq 450$ & $\mathrm{PLT}>450$ \\
\hline $\mathrm{EF} 2$ & $\mathrm{EF} \leq 50$ & $\mathrm{EF}>50$ & - \\
\hline Age2 & - & $\begin{array}{c}\text { if male \& } \\
\text { Age } \leq 45 \\
\text { or if female \& } \\
\text { Age } \leq 55\end{array}$ & $\begin{array}{c}\text { if male \& } \\
\text { Age }>45 \\
\text { Or if female } \\
\& \\
\text { Age }>55\end{array}$ \\
\hline $\mathrm{BP} 2$ & $\mathrm{BP}<90$ & $90 \leq \mathrm{BP} \leq 120$ & $\mathrm{BP}>120$ \\
\hline PR2 & $\mathrm{PR}<60$ & $60 \leq \mathrm{PR} \leq 100$ & $\mathrm{PR}>100$ \\
\hline Neut 2 & - & Neut $\leq 65$ & Neut $>65$ \\
\hline TG2 & - & $\mathrm{TG} \leq 200$ & $\mathrm{TG}>200$ \\
\hline $\begin{array}{c}\text { Function } \\
\text { Class2 }\end{array}$ & - & 1 & $2,3,4$ \\
\hline HR2 & $\mathrm{HR}<60$ & $60 \leq \mathrm{HR} \leq 100$ & $\mathrm{HR}>100$ \\
\hline $\begin{array}{l}\text { Total } \\
\text { chol } 2\end{array}$ & $\begin{array}{c}200 \leq \text { Total } \\
\text { chol } \leq 240 \\
\text { is desirable }\end{array}$ & Total chol<200 & $\begin{array}{c}\text { Total chol > } \\
240\end{array}$ \\
\hline Lymph2 & $\begin{array}{c}\text { Lymph }< \\
20\end{array}$ & $20 \leq$ Lymph $\leq 40$ & Lymph > 40 \\
\hline
\end{tabular}


Table (3) and Table (4) present the discretization of some of the most important features obtained from ECG that decrease the data.

Table 3: Discretization of ECG features

\begin{tabular}{|c|c|c|c|}
\hline Feature & Short & Normal & Long \\
\hline $\begin{array}{c}\text { PR } \\
\text { Interval }\end{array}$ & $\begin{array}{c}\text { PR } \\
\text { Interval } \\
<0.12\end{array}$ & $\begin{array}{c}0.12 \leq \text { PR Interval } \leq \\
0.20\end{array}$ & $\begin{array}{c}\text { PR } \\
\text { Interval } \\
>0.20\end{array}$ \\
\hline $\begin{array}{c}\text { QT } \\
\text { Interval } \\
2\end{array}$ & $\begin{array}{c}\text { QT } \\
\text { Interval } \\
<0.35\end{array}$ & $0.35 \leq$ QT Interval $\leq$ & $\begin{array}{c}\text { QT } \\
\text { Interval } \\
>0.45\end{array}$ \\
\hline
\end{tabular}

Table 4: Discretization of QRS feature

\begin{tabular}{|l|c|c|c|}
\hline Feature & Narrow & Normal & Wide \\
\hline $\mathrm{QRS} 2$ & $\mathrm{QRS}<0.08$ & $0.08 \leq \mathrm{QRS} \leq 0.12$ & $\mathrm{QRS}>0.12$ \\
\hline
\end{tabular}

\section{3- Method}

Extraction of effective features for classifying the risk of patients under CRT is important for improving CRT response. This research is aimed at evaluating the prediction of CRT response by analysis of clinical, laboratory, and ECG data. The most important measures in this research are using the most well-known machine learning algorithms for creating a prediction model and proposing a comparative method for optimal classification on the proposed feature vector. Fig. (1) presents the overall phases of the research for prediction of CRT response in patients, prioritizing the variables from the experts' viewpoints, and their effectiveness in prediction.

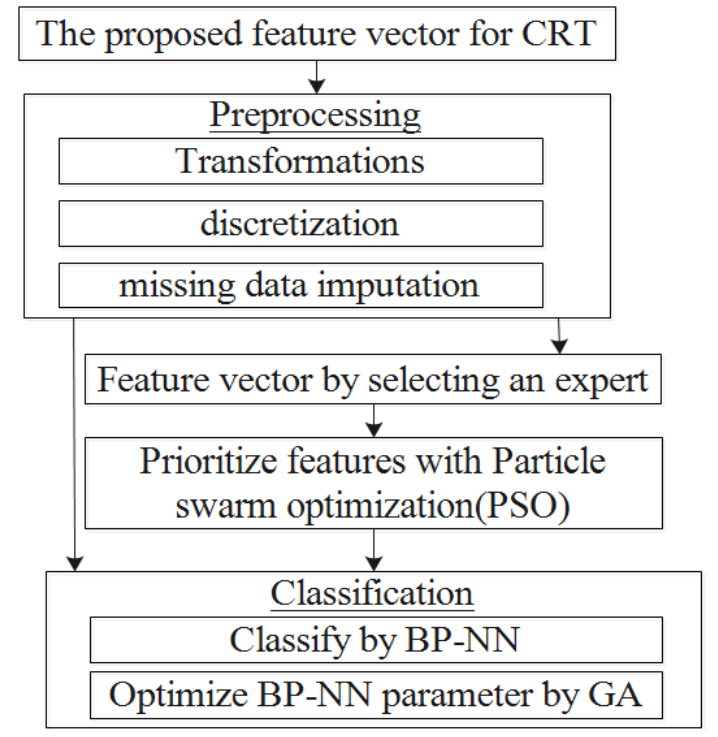

Fig. 1: Overall research method for recognizing the priorities of prediction variables
In the preprocessing section, three operations of transformation, discretization, and imputation are performed to improve the input data. The transformation operation transforms some qualitative attributes into quantitative ones. For example, the Device_Type attribute has two types of CRT-P and CRT-D, or the Rhythm attribute has two types of AF and Sin, which can take zero and one value, respectively. Quantification provides the conditions for the use of many machine learning algorithms. In discretization, the goal is to reduce data. Many variables have large numerical dispersions that reduce the efficiency of the classifier in detection. By discretizing the attributes based on the medical doctor's knowledge, more favorable conditions can be created based on clinical trial intervals in decision making for the classifier. The aim is to reduce the data and easier decision making for the algorithm. The predictive imputation process can prevent presenting sets with lost data. In this dataset, very little data is lost due to incomplete patient records. The results of the predicted imputation were evaluated based on the patient's other data with the supervision of a medical doctor. The imputation process was performed by prediction by the K-Nearest Neighbor (K-NN) algorithm with one neighborhood. The subject of the number of neighborhoods in producing a logical answer was examined from the perspective of a medical doctor. It revealed that the higher number of neighborhoods provide inappropriate answers for prediction due to the distance of neighbors from the studied samples.

\section{3-1- Backpropagation of Error Neural Network}

In the following sections, the most important used algorithms are briefly introduced and then, the method of calculating efficiency is described. The architecture of the back propagation neural networks (BP-NN) is the most popular model for complex and multilayer networks. The training process is usually done by delta rule that starts with the calculated difference between the real outputs and the desired outputs. The neural network determines the primary prediction by using Eq. (1), and in the case of $y \neq$ Target, the weights become updated based on the error value [28].

$y=f\left(b+\sum_{i=1}^{n} w_{i} x_{i}\right)$

In this study, a multilayer perceptron back propagation neural network with a hidden layer was presented. Since 
some features lacked information and some features were not computational, they were removed from the set for analysis. 61 features were given to the neural network input and 34 nodes were considered as hidden layer nodes for greater productivity. This number has been set based on the logic of adequate accuracy and fewer nodes in the hidden layer. At the beginning of the experiment, the hidden layer nodes were equal to 61 nodes and the number 34 was selected with different iterations, which the algorithm offers better speed without reducing the classification accuracy. At the network output, two nodes were placed to determine the status of cardiac synchronization treatment which the prediction results are determined based on the reliability of each output node.

\section{3-2- Particle Swarm Optimization (PSO)}

Particle swarm optimization begins with a random initialization to particles population. These particles show the dataset properties of the research. The value of each property is random to adjust the initial position of the particle while the initial particle velocity is set to zero. The particle density should not exceed the maximum value of each property of the sample dataset. After initialization, each particle is evaluated with an evaluator function to find the value of pbest. From the value of pbest obtained, a value is selected as the best value of gbest. Then, the velocity and position of each particle are updated using Equation 2 and Equation 3.

$$
\begin{aligned}
& V i(t)=V i(t-1)+c 1 r 1(x p i-x i) \\
& +x 2 r 2(x g-x i) \\
& x i(t)=x i(t-1)+v i(t)
\end{aligned}
$$

Here $\mathrm{v}$ is the velocity, $\mathrm{x}$ is the particle position, $\mathrm{c}$ is the learning rate, and $r$ is a random number between zero and one and $v i(t-1)$ inertia, which forces the particle to move in the same direction as before. $\operatorname{cr} 2 r 2(x g-x i)$ is a social expression that forces particles to move to the best position of all particles. The evaluation process by proportional function, position, and density update is performed as an iterative operation until the expected termination conditions are met. If the evaluation performance tends to improve very slightly, convergence is obtained whose output is a weight value for each property. In fact, the higher weight indicates the effect of the property to achieve a better position of the particle [29]. In the research method, the obtained weights are used to prioritize the vector recommended by the medical doctor.

\section{3-3- Measuring Efficiency}

Accuracy of a classifier is the probability of accurate prediction of the class of samples without a label. The experimental samples for prediction of CRT response are classified in one of the four variables below; in other words, accuracy criteria for binary classification can be explained in four categories:

- $\mathbf{T P}$ or positive true is the number of patients who faced with no CRT response in a true class.

- $\mathbf{T N}$ or true negative is the number of patients who responded to CRT in true class.

- $\quad \mathbf{F P}$ or false positive is the number of patients who faced with no CRT response in a false class.

- $\quad \mathbf{F N}$ or false negative is the number of patients who responded to CRT in a false class.

Classifier sensitivity or inclusiveness is determined by true positive division into the total positive true and false negative samples. Eq. (4) presents the percentage of reliability of no CRT response.

Sensitivity $=\frac{T P}{T P+F N}$

However, a specificity that is a kind of accuracy is determined by dividing true negatives by the total true negative and false positive samples. Actually, Eq. (5) indicates the way of determining the appropriate cases of CRT response.

Specificity $=\frac{T N}{T N+F P}$

The ability of accurate detection of patients needing and not needing CRT to become possible by accuracy metric. In order to evaluate the accuracy, the ratio of all the true predictions of response and no response to the total prediction is considered. Eq. (6) indicates this ratio.

Accuracy $=\frac{T P+T N}{T P+F P+T N+F N}$

For measuring F-measure, F score, or F1 score, a harmonic average of two scores multiplied with two is defined that is proposed for the balance of sensitivity and specificity. Eq. (7) indicates the calculation of the F score.

$F=2 \times \frac{\text { Sensitivity } \times \text { Specificity }}{\text { Sensitivity }+ \text { Specificity }}$

$\mathrm{K}$-fold cross-validation (KCV) is a technique for precise evaluation in which, the created model is applied on rotated test sets for $\mathrm{k}$ times. In the proposed method, the $\mathrm{k}$ value is considered equal to 10 . Measuring precision affects model selection. 


\section{4- Experimental Results}

In this section, five machine learning algorithms are applied for prediction of the condition after implanting the device. Each of these algorithms will have different performance in pattern recognition and they will provide different predictions of CRT response or no response.

\section{4-1- The Results of The Primary Classification}

The tested algorithms include NB classifier, SVM, random forest, K-NN, and neural network algorithm. Since no CRT response is determined as the positive class, improvement of the total accuracy of classification mainly depends on the accuracy of the negative class. In order to get the highest classification accuracy, algorithms try to maximize the accuracy of this class and it will lead to a decrease in the accuracy of the positive class. Random Forest has the highest sensitivity rate, but it cannot have a high rate of accuracy due to low specificity. Three algorithms of neural network, support vector machine, and k-nearest neighbors have respectively the highest specificity. These three algorithms present the specificity criteria in a close range. So, they play a determinative role in selecting a better algorithm. Neural network provides a more optimal condition due to having the highest specificity compared with the other two algorithms. The low F-measure indicates the low sensitivity of the algorithms and it suggests that prediction of no CRT response has a low rate in the research dataset. Although high specificity indicates the quality of prediction in recognition of positive CRT response after total accuracy F-measure should be carefully investigated for comparing efficiency rates. In the primary test, a decision tree was selected, but a classifier similar to the random forest was replaced for that due to lack of sensitivity. In the primary evaluation, Table (5) presents the efficiency of error back propagation neural networks (BP-NN).

Table 5: The results of classifying the five algorithms in terms of CRT

\begin{tabular}{|c|c|c|c|c|c|}
\hline Performance & $\boldsymbol{N B}$ & $\boldsymbol{K}-\boldsymbol{N N}$ & $\begin{array}{c}\boldsymbol{B P}- \\
\boldsymbol{N N}\end{array}$ & $\boldsymbol{S V M}$ & $\boldsymbol{R F}$ \\
\hline Accuracy & 69.36 & 71.73 & $\underline{\mathbf{7 3 . 2 4}}$ & 66.54 & 68.9 \\
\hline F-Measure & 17.95 & 19.22 & 9.68 & 12.5 & $\underline{\mathbf{1 9 . 7 5}}$ \\
\hline Sensitivity & 18.93 & 18.93 & 8.21 & 13.93 & $\underline{\mathbf{2 1 . 7 9}}$ \\
\hline Specificity & 80.32 & 83.13 & $\underline{\mathbf{8 7 . 2 4}}$ & 78.05 & 79.14 \\
\hline
\end{tabular}

\section{4-2- Achieving a Higher Performance}

With regard to the results of comparing the algorithms, it can be expected that parameter optimization of neural network models causes higher efficiency in the classification process. In the following of the test, two further neural networks including single layer perceptron and learning vector quantization neural network (NNLVQ) were compared. Fig. (2) indicates that BP-NN parametric optimization significantly improves the total accuracy of detection. However, consequences of this optimization lead to a decrease in sensitivity. The genetic algorithm produces the initial population by producing different chromosomes from different parameters of the neural network algorithm. The proportional function evaluates the dataset based on the parameters of each chromosome. Selected chromosomes are passed on to the next generation and in the next generation, new compounds are made using crossovers. In this study, the mutation is not used to produce compounds. Ten percent of each generation's choices have been passed on to the next generation, and in 90 percent the intersections have been used. Finally, if there is no improvement in 3 generations or continuity for up to 50 generations, the exit from the algorithm will be done and the chromosome will show the optimal parameters of the algorithm with the highest accuracy of classification. Ultimately, if there is no improvement in 3 generations or continuity for up to 50 generations, the exit from the algorithm will be done and the chromosome with the highest accuracy of classification will show the optimal parameters of the algorithm.

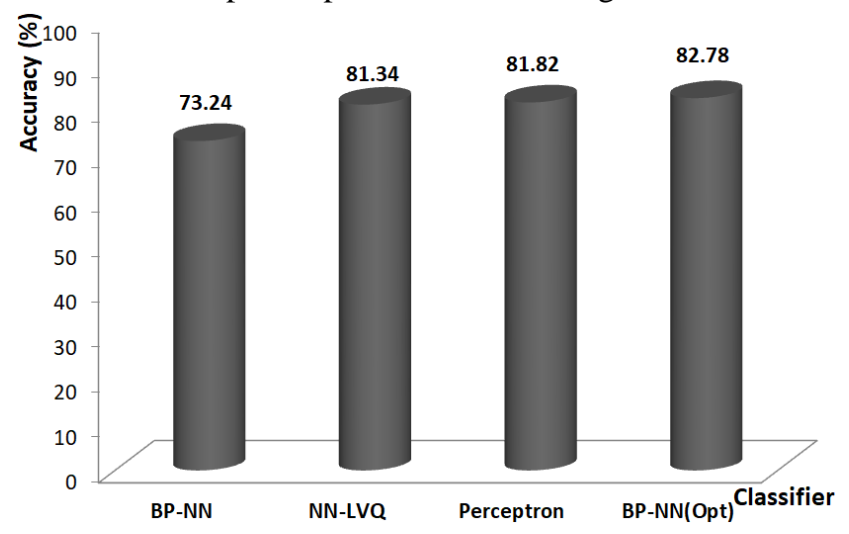

Fig. 2: The results of improvement of classification by neural network models

The two parameters of learning rate $(0.561)$ and momentum (0.923) of the neural network were improved when being trained by genetic algorithm. Also, the other 
two parameters of epoch number (1000) and error epsilon were set manually.

All the models of neural network indicated more optimal conditions than the studied machine learning algorithms. Therefore, more improvement in neural network models can be expected. Through separating a subset of the no response samples with the minimum number of samples from a different class, an appropriate model can be created for better recognition of this class by using BP-NN algorithm. Determining the effect of the variable in the prediction of CRT response on 9 important features from the doctor's viewpoint can indicate the priority of selecting the features as an optimization operation. Two features in the subset of the selected features do not have information gain (IG). So according to Table (6), the analysis continues without considering CHF.

Higher weights do not necessarily refer to the effect of a feature on prediction. However, zero weight refers to a lack of information variety for decision making. Creating various combinations of features by particle swarm optimization (PSO) algorithm and using 8 features can be an effective solution for recognizing the effect of variables. PSO algorithm was used for general minimization and weighting the features. First, the algorithm randomly created a group of particles. Then, it searched for the optimal solution by updating generations. Two possible local and global locations of the particle are effective in updating particles.

Table 6: Weighting the features with information gain (IG)

\begin{tabular}{|c|c|c|}
\hline Attribute & Weight & Select \\
\hline CHF & 0 & N \\
\hline Lung_rales & $7.80 \mathrm{E}-04$ & $\mathrm{Y}$ \\
\hline QRS_Duration2 & $2.11 \mathrm{E}-03$ & $\mathrm{Y}$ \\
\hline Dyspnea & $5.44 \mathrm{E}-03$ & $\mathrm{Y}$ \\
\hline Cr2 & $6.79 \mathrm{E}-03$ & $\mathrm{Y}$ \\
\hline Function_Class & $9.13 \mathrm{E}-03$ & $\mathrm{Y}$ \\
\hline BUN2 & $1.01 \mathrm{E}-02$ & $\mathrm{Y}$ \\
\hline LV_EF2 & $1.23 \mathrm{E}-02$ & $\mathrm{Y}$ \\
\hline Number_of_fragment & $1.24 \mathrm{E}-02$ & $\mathrm{Y}$ \\
\hline
\end{tabular}

BP-NN classifier was selected as the fitness function with optimal parameters, the population of 50 , the generation number of 100 , and the exclusion condition in the case of no improvement was considered equal to 2. Fig. (3), presents the results of weighting and the importance of each feature related to CRT response.

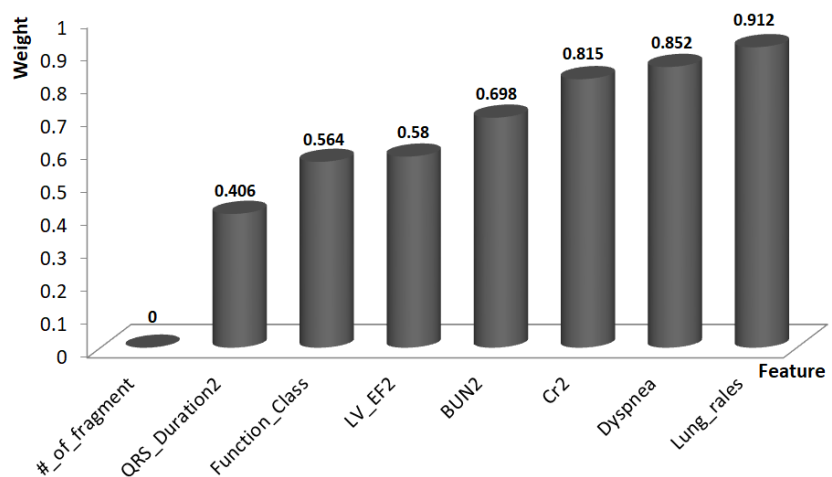

Fig. 3: Prioritization of variables based on the influence coefficient of the PSO algorithm

So, feature weighting method is about searching for the weight of a feature by PSO in order to specify its effect on output results.

\section{4-3- Discussion}

After selecting the CRT candidate patients, there are many challenges and problems before a successful treatment. In HF patients, CRT improves the quality of life and decreases the death rate. However, making a decision about different issues such as CRT heart pacemaker [30], and post-surgery suggestions [31] are effective in the improvement of treatment. Classification process provides the possibility of predicting CRT response before treatment of a patient in a probabilistic manner. Among the studied algorithms, neural network models indicated a higher efficiency. By setting the parameters for reaching a higher precision in $\mathrm{NN}$ training, it was found that the maximum increase in specificity leads to a maximum decrease in sensitivity and consequently, decreases of Fmeasure. So, the major technical challenge of this dataset is improving sensitivity without decrease of the total precision. In this paper, the PSO algorithm that is one of the collective intelligence algorithms was used for prioritizing the variables. Among the 8 important features selected by the doctor, Lung rales and Dyspnea were the most effective features. Since most of the patients faced with no response, they were considered as $\mathrm{CHF}=1$; also since the number of fragments was predictable regarding the existence of other independent variables, they were less important than other features in decision making. 


\section{5- Conclusions}

In this paper, we have presented two goals of a selection of appropriate candidates for CRT and machine prioritization of the feature vector limited by the doctor. In the evaluation of the 9 variables considered important by the doctor in making a decision about prediction, prioritizes of 8 features were determined by the PSO algorithm. The two variables of Lung rales and Dyspnea have the highest effectiveness in the prediction of no CRT response. The improvement in class separability resulted in a significant increase in individual class accuracy achieved by the ANN classifier, and an overall accuracy of $82.78 \%$ was reached. The future efforts will be aimed at evaluating the efficiency and the smaller feature vector and solving the problem of low sensitivity in the prediction of CRT response. According to the comparison of the proposed machine learning algorithms and advantages of neural network models in classifying the research dataset, and also regarding the possibility of extending neural network models and optimizing the various parameters of the neural network, it can be concluded that development of neural network models can be promising.

\section{Compliance with ethical standards}

Conflict of interest: The authors declare that they have no conflict of interest.

Ethical approval: Our research was approved by the Behavioral Research Ethics Board at the Islamic Azad University, Rasht Branch, Guilan, Iran.

\section{References}

[1] R. C. Deo, "Machine learning in medicine", Circulation, vol. 132, no. 20, 2015, pp. 1920-1930.

[2] H. Assodiky, I. Syarif, and T. Badriyah, "Deep Learning Algorithm for Arrhythmia Detection", International Electronics Symposium on Knowledge Creation and Intelligent Computing (IES-KCIC), 2017, pp. 26-32.

[3] A. K. Flügge, K. Wasmer, S. Orwat, H. Abdul-Khaliq, P. C. Helm, U. Bauer, H. Baumgartner, and G. P. Diller, "Cardiac resynchronization therapy in congenital heart disease: Results from the German National Register for Congenital Heart Defects," International Journal of Cardiology, vol. 273, 2018, pp. 108-111.

[4] J. Rickard, A. Cheng, D. Spragg, S. Bansal, M. Niebauer, B. Baranowski, D. J. Cantillon, P. J. Tchou, R. A. Grimm, W. H. W. Tang, B. L. Wilkoff, and N. Varma, "Durability of the survival effect of cardiac resynchronization therapy by level of left ventricular functional improvement: Fate of "nonresponders"," Heart Rhythm, vol. 11, no. 3, 2014, pp. 412-416.

[5] H. X. Niu, W. Hua , F. Z. Wang, S. Zhang, K. P. Chen, and $\mathrm{X}$. Chen, "Complications of cardiac resynchronization therapy in patients with congestive heart failure," Chinese Medical Journal, vol. 119, no. 6, 2006, pp. 449-453.

[6] C. Leclercq, H. Burri, A. Curnis, P. P. Delnoy, C. A. Rinaldi, J. Sperzel, K. Lee, C. Cohorn, and B. Thibault, "Rationale and design of a randomized clinical trial to assess the safety and efficacy of multipoint pacing therapy: MOre REsponse on Cardiac Resynchronization Therapy with MultiPoint Pacing (MORE-CRT MPP-PHASE II)," American Heart Journal, vol. 209, 2019, pp. 1-8.

[7] H. Huang, L. Shen, R. Zhang, F. Makedon, B. Hettleman, and J. Pearlman, "Cardiac Motion Analysis to Improve Pacing Site Selection in CRT," Academic Radiology, vol. 13, no. 9, 2006, pp. 1124-1134.

[8] M. M. Kalscheur, R. T. Kipp, M. C. Tattersall, C. Mei, K. A. Buhr, D. L. DeMets, M. E. Field, L. L. Eckhardt, and C. D. Page, "Machine Learning Algorithm Predicts Cardiac Resynchronization Therapy Outcomes: Lessons From the COMPANION Trial," Circ Arrhythm Electrophysiol, vol. 11, no. 1, 2018, pp. 1-11.

[9] S. Giffard-Roisin, H. Delingette, T. Jackson, J. Webb, L. Fovargue, J. Lee, C. A. Rinaldi, R. Razavi, N. Ayache, and M. Sermesant, "Transfer Learning From Simulations on a Reference Anatomy for ECGI in Personalized Cardiac Resynchronization Therapy," IEEE Transactions on Biomedical Engineering, vol. 66, no. 2, 2019, pp. 343-353.

[10] K. Howe, P. Gladding, N. James, C. Prabhakar, L. Dawson, A. Gavin, and T. Schlegel, "Predicting CRT Response Using Machine Learning Analysis of Pre-Implant ECG Data," Heart, Lung and Circulation, vol. 26, no. 1, 2017, pp. S25S26.

[11] I. Kononenko, "Machine learning for medical diagnosis: history, state of the art and perspective," Artificial Intelligence in Medicine, vol. 23, no. 1, 2001, pp. 89-109.

[12] P. K. Chao, C. L. Wang, and H. L. Chan, "An intelligent classifier for prognosis of cardiac resynchronization therapy based on speckle-tracking echocardiograms," Artificial Intelligence in Medicine, vol. 54, no. 3, 2012, pp. 181-188.

[13] M. Fedorco, A. Bulava, P. Šantavý, A. Mokráček, V. Lonský, L. Dušek, and M. Táborský, "Middle-term stability of epicardial left ventricular electrodes for cardiac resynchronization therapy," Cor et Vasa, vol. 59, no. 6, 2017, pp. e530-e539.

[14] R. Zhang, S. Ma, L. Shanahan, J. Munroe, S. Horn, and S. Speedie, "Discovering and identifying New York heart association classification from electronic health records," BMC Medical Informatics and Decision Making, vol. 18, no. 2, 2018, pp. 5-13.

[15] N. M. Hawkins, M. C. Petrie, M. I. Burgess, and J. J.V. McMurray, "Selecting Patients for Cardiac Resynchronization Therapy: The Fallacy of Echocardiographic Dyssynchrony," Journal of the American College of Cardiology, vol. 53, no. 21, 2009, pp. 1944-1959.

[16] J. Rickard, H. Michtalik, R. Sharma, Z. Berger, E. Iyoha, A. R. Green, N. Haq, and K. A. Robinson, "Predictors of response to cardiac resynchronization therapy: a systematic review," International Journal of Cardiology, vol. 225, 2016, pp. 345-352.

[17] M. Tokodi, W. Schwertner, P. Perge, A. Kosztin, B. Lakatos, S. Shrestha, A. Kovacs, and B. Merkely, "Unsupervised machine learning algorithm to identify high and low risk patients following crt implantation," Journal of 
the American College of Cardiology, vol. 71, no. 11, 2018, pp. 947-948.

[18] M. Loutfi, M. Nawar, S. Eltahan, and A. A. Elhoda, "Predictors of response to cardiac resynchronization therapy in chronic heart failure patients," The Egyptian Heart Journal, vol. 68, no. 4, 2016, pp. 227-236.

[19] A. Grimaldi, E. Z. Gorodeski, and J. Rickard, "Optimizing Cardiac Resynchronization Therapy: an Update on New Insights and Advancements," Current Heart Failure Reports, vol. 15 , no. 3,2018 , pp. 156-160.

[20] O. Adegbala, O. Olagoke, A. Adejumo, E. Akintoye, A. Oluwole, P. Alebna, K. Williams, R. Lieberman, and L. Afonso, "Incidence and outcomes of cardiac tamponade in patients undergoing cardiac resynchronization therapy," International journal of cardiology, vol. 272, 2018, pp. 137141.

[21] C. J. Plummer, C. M. Frank, Z. Bári, Y. S. Al Hebaishi, R. N. Klepfer, R. W. Stadler, S. Ghosh, S. Liu, and S. Mittal, "A novel algorithm increases the delivery of effective cardiac resynchronization therapy during atrial fibrillation: The CRTee randomized crossover trial," Heart Rhythm, vol. 15, no. 3,2018 , pp. 369-375.

[22] E. Thomas, D. Toth, T. Kurzendorfer, K. Rhode, and P. Mountney, "Mechanical Activation Computation from Fluoroscopy for Guided Cardiac Resynchronization Therapy," in 2018 40th Annual International Conference of the IEEE Engineering in Medicine and Biology Society $(E M B C), 2018$, pp. 592-595.

[23] D. J. van Veldhuisen, A. H. Maass, S. G. Priori, P. Stolt, I. C. van Gelder, K. Dickstein, and K. Swedberg, "Implementation of device therapy (cardiac resynchronization therapy and implantable cardioverter defibrillator) for patients with heart failure in Europe: changes from 2004 to 2008," European Journal of Heart Failure, vol. 11, no. 12, 2009, pp. 1143-1151.

[24] G. Boriani, M. Ziacchi, M. Nesti, A. Battista, F. Placentino, V. L. Malavasi, I. Diemberger, and L. Padeletti, "Cardiac resynchronization therapy: How did consensus guidelines from Europe and the United States evolve in the last 15 years?," International journal of cardiology, vol. 261, 2018, pp. 119-129.

[25] J. Gorcsan, C. P. Anderson, B. Tayal, M. Sugahara, J. Walmsley, R. C. Starling, and J. Lumens, "Systolic Stretch Characterizes the Electromechanical Substrate Responsive to Cardiac Resynchronization Therapy," JACC: Cardiovascular Imaging, vol. 12, no. 9, 2018, pp. 1741-1752.

[26] M. A. Baturova, V. Kutyifa, S. McNitt, B. Polonsky, S. Solomon, J. Carlson, W. Zareba, and P. G. Platonov, "Usefulness of Electrocardiographic Left Atrial Abnormality to Predict Response to Cardiac Resynchronization Therapy in Patients With Mild Heart Failure and Left Bundle Branch Block," The American Journal of Cardiology, vol. 122, no. 2, 2018, pp. 268-274.

[27] D. Peressutti, W. Bai, T. Jackson, M. Sohal, A. Rinaldi, D. Rueckert, and A. King, "Prospective Identification of CRT Super Responders Using a Motion Atlas and Random Projection Ensemble Learning," in International Conference on Medical Image Computing and Computer-Assisted Intervention, 2015, vol. 9351, pp. 493-500.
[28] S. Shanmuganathan, "Artificial Neural Network Modelling: An Introduction," in Studies in Computational Intelligence, vol. 628, 2016, pp. 1-14.

[29] A. Dongoran, S. Rahmadani, M. Zarlis, and S. Zakarias, "Feature weighting using particle swarm optimization for learning vector quantization classifier," in 2nd International Conference on Computing and Applied Informatics, 2017 vol.978, pp.1-6.

[30] D. Weber, M. Koller, D. Theuns, S. Yap, M. Kühne, C. Sticherling, T. Reichlin, T. Szili-Torok, S. Osswald, and B. Schaer, "Predicting defibrillator benefit in patients with cardiac resynchronization therapy: A competing risk study," Heart Rhythm, vol. 16, no. 7, 2019, pp. 1057-1064.

[31] F. Leyva, A. Zegard, O. N Okafor, J. de Bono, D. McNulty, A. Ahmed, H. J Marshall, D. Ray, and T. Qiu, "Survival after cardiac resynchronization therapy: results from 50084 implantations," EP Europace, vol. 21, no. 5, 2019, pp. 754762.

Mohammad Nejadeh received the B.S. degree in Computer Engineering from Islamic Azad University, Lahijan Branch, Iran, and M.S. degree in Information Technology from Guilan University , Iran. Currently $\mathrm{He}$ is Ph.D. Candidate in Islamic Azad University, Rasht Branch, Iran. His research interests include Information Technology, Network Security, Artificial Intelligence, Computer Engineering Sciences.

Peyman Bayat received the M.Sc. degree from Islamic Azad University, Arak Branch, Iran, and the Ph.D. degree in computer engineering from UPM University, Malaysia. He is currently an Assistant Professor with the Faculty of Computer Engineering, Islamic Azad University, Rasht Branch, Iran. His main research interests include distributed systems, Artificial Intelligence.

Jalal Kheirkhah received the Specialty Doctor in Cardiology in Shahid Beheshti University of Medical Sciences, Tehran, Iran, in 2004 and the Fellowship of Clinical Cardiac Electrophysiology in Tehran University of Medical Sciences, Tehran, Iran in 2011. His area research interests include Cardiac Electrophysiology and Cardiovascular Imaging.

Hassan Moladoust received the M.S. and Ph.D degrees in Medical Physics from Tarbiat Modares University, Tehran, Iran, in 2001 and 2008; respectively. His area research interests include Cardiovascular Imaging, Signal and Image analysis. 